\section{The Big-Five Personality Traits and Quality of Life in Elderly Malaysian Patients with Diabetes Mellitus: A Cross-Sectional Study}

\author{
WOON LSC ${ }^{1}$, GOSSE PJ ${ }^{2}$, KAUNISMAA ES ${ }^{2}$, MAINLAND RL $^{2}$, \\ ARUN R ${ }^{3}$, HATTA $S^{1}$ \\ ${ }^{1}$ Department of Psychiatry, Faculty of Medicine, Universiti Kebangsaan Malaysia Medical \\ Centre, Jalan Yaacob Latif, Bandar Tun Razak, 56000 Cheras, Kuala Lumpur, Malaysia \\ ${ }^{2}$ Faculty of Medicine, University of Toronto, 1 King's College Circle, Toronto, Ontario, \\ M5S 1A8, Canada \\ ${ }^{3}$ Centre for Addiction and Mental Health, Faculty of Medicine, University of Toronto, 1 \\ King's College Circle, Toronto, Ontario, M5S 1A8, Canada
}

\begin{abstract}
ABSTRAK
Walaupun penyakit kencing manis sering dijumpai dalam kalangan warga emas serta memberi kesan yang ketara terhadap kualiti hidup (QOL) seseorang, kita masih tidak mengetahui secara jelas cara trait personaliti mempengaruhi QOL. Kajian keratan rentas ini yang dijalankan di Pusat Perubatan Universiti Kebangsaan Malaysia adalah bertujuan untuk menentukan hubung kait di antara trait personaliti dan $Q O L$ dalam kalangan warga emas (berumur $\geq 60$ tahun) yang mengalami penyakit kencing manis. Maklumat sosio-demografik dan klinikal telah dikumpulkan. QOL diukur dengan soal sedilik WHO Quality of Life-BREF (WHOQOL-BREF) sementara trait personaliti pula dinilai dengan Big Five Inventory (BFI). Kemurungan dan keresahan diukur dengan pengujian soal selidik Beck Depression Inventory-II (BDIII) dan Generalized Anxiety Disorder 7-item (GAD-7) masing-masing dan diambil dalam analisa statistik. Terdapat sebanyak 170 peserta kajian yang melibatkan diri (umur median = 69.0 tahun; IQR: 65.0-73.0; dengan 51.2\% lelaki). Menggunakan model 'stepwise linear regression', didapati skor kehematan $(\beta=0.156 ; P=0.044)$ dan skor neurotisisme yang negatif ( $\beta=-0.176 ; P=0.028$ ) amat berkait dengan $Q O L$ yang baik di dalam domain kesihatan fizikal. Sementara nilai skor ekstraversi $(\beta=0.209 ; P=0.001)$ dan skor kehematan yang tinggi $(\beta=0.248 ; P<0.001)$ pula amat berkait dengan $Q O L$ yang baik di dalam domain kesihatan psikologi. Ketinggian skor kepersetujuan $(\beta=0.286 ; P<0.001)$ amat berkait rapat dengan $Q O L$ yang baik di dalam domain perhubungan sosial. Akhirnya, skor kepersetujuan yang tinggi $(\beta=0.327 ; P<0.001)$ dan darjah neurotisme $(\beta=-0.223 ; P=0.001)$ didapati amat
\end{abstract}

Address for correspondence and reprint requests: Luke Sy-Cherng Woon. Department of Psychiatry, Faculty of Medicine, Universiti Kebangsaan Malaysia Medical Centre, Jalan Yaacob Latif, Bandar Tun Razak, 56000 Cheras, Kuala Lumpur, Malaysia. Tel: +603-91456423 Email: lukewoon@ukm.edu.my 
berkait dengan QOL yang baik di dalam domain persekitaran. Trait personaliti berkait rapat dengan semua domain QOL di kalangan pesakit warga emas yang mengalami penyakit kencing manis. Personaliti sebelum jatuh sakit (premorbid personality) mungkin mempunyai peranan penting di dalam mempengaruhi kesan penyakit kencing manis terhadap kehidupan pesakit warga emas.

Kata-kunci: diabetes mellitus, kesihatan mental, kualiti hidup, personaliti, warga tua

\section{ABSTRACT}

While diabetes mellitus is highly prevalent in the elderly population with significant impact on quality of life (QOL), we yet to know much about how personality traits affect QOL in this patient population. A cross-sectional study was conducted at Universiti Kebangsaan Malaysia Medical Centre with the aim to determine the relationship between personality traits and QOL among the elderly (aged $\geq 60$ years) with diabetes mellitus. Sociodemographic and clinical information were obtained. QOL was assessed using the WHO Quality of Life-BREF (WHOQOLBREF) questionnaire and personality traits were assessed using the Big Five Inventory (BFI) questionnaire. Depression and anxiety were measured with Beck Depression Inventory-II (BDI-II) and Generalized Anxiety Disorder 7-item (GAD-7) scale, respectively, and controlled for in all analyses. There were in total 170 study participants (median age $=69.0$ years; IQR: 65.0-73.0; 51.2\% male). In stepwise linear regression models, higher conscientiousness scores $(\beta=0.156 ; P=0.044)$ and lower neuroticism scores $(\beta=-0.176 ; P=0.028)$ were associated with greater $\mathrm{QOL}$ in the physical health domain. Higher extraversion scores $(\beta=0.209 ; P=0.001)$ and higher conscientiousness scores $(\beta=0.248 ; P<0.001)$ were associated with greater $\mathrm{QOL}$ in the psychological health domain. Higher agreeableness scores $(\beta=0.286$; $P<0.001)$ were associated with greater QOL in the social relationship domain. Finally, higher agreeableness scores $(\beta=0.327 ; P<0.001)$ and lower neuroticism scores $(\beta=-0.223 ; P=0.001)$ were associated with greater $\mathrm{QOL}$ in the environment domain. Personality traits were closely associated with all domains of QOL among elderly patients with diabetes mellitus. Premorbid personality may have important role in moderating the impact of diabetes mellitus on the lives of elderly patients.

Keywords: diabetes mellitus, elderly, mental health, personality, quality of life

\section{INTRODUCTION}

Diabetes mellitus (DM) is a common disease associated with significant microvascular and macrovascular complications (Fowler 2008). As optimal glycaemic control is crucial in preventing these complications, maintaining glycaemic control is essential. This can only be achieved 
through the combination of rigorous lifestyle modifications and pharmacotherapy. In this respect, elderly patients with diabetes require different management approaches than their younger counterparts. Geriatric populations face specific challenges related to health, including frailty, functional limitation, changes in cognitive ability and mental health, as well as increased dependency on others for care (Gregg et al. 2002). With this complexity of care comes a need to better understand the psychosocial factors that influence the health of this population.

While certain social, clinical, and psychological factors that impact DM management have been highlighted in previous studies, the influence of personality traits on the quality of life (QOL) in elderly patients with diabetes has not been adequately explored. The Big Five Model of personality includes the following domains i.e., extraversion, agreeableness, neuroticism, conscientiousness, and openness to experience (Costa \& McCrae 1990). These traits are believed to remain stable across time. In a large nationally representative sample of adults in Australia, personality, as measured according to the Big Five Model, changed minimally across four years (Cobb-Clark \& Schurer 2012). Another study followed individuals over 45-years and found stability in the domains of neuroticism, conscientiousness, and openness to experience across time (Soldz \& Vaillant 1999). As diabetes is a chronic condition, it is important to consider the impact of stable factors such as personality on long-term disease management in these individuals.

Personality may impact disease perception and QOL in individuals with diabetes. One study conducted in Israel among older adults with type 2 DM examined the effect of personality traits on the relationship between subjective health (a patient's perception of their health) and objective health (measured by various health outcomes). They found that participants with higher neuroticism scores had stronger associations between subjective and objective health, suggesting that this trait impacts how a patient views their illness (Elran-Barak et al. 2019). A review by Schimmack et al. (2004) explored the relationship between QOL (defined as Health-Related Quality of Life and Life Satisfaction) and personality traits (defined by the five-factor model). They found a significant positive association between extraversion and life satisfaction. The World Health Organization has further developed the general concept of QOL into four different aspects: physical health, psychological health, social relationships, and environment (WHOQOL Group 1998). A study by Yamaoka et al. (1998) found that extraversion correlated positively with health related QOL in both healthy and cancer populations. All four of these domains may be impacted by the disease burden associated with DM as well as the personality traits of the individual.

Malaysia is a middle-income country with a unique socio-cultural landscape and a high incidence rate of DM reported to be at $20.8 \%$ in 
2011 (Hussein et al. 2015). In addition, the country has a rapidly ageing population; by 2040, it is estimated that $15 \%$ of the population will be above the age of 65 (Department of Statistics Malaysia 2016). As the incidence of DM continues to rise with the average age of the population continues to increase, so will the related costs to the Malaysian healthcare system. Thus, there is a great need in expanding our understanding of psychosocial factors affecting treatment outcomes among the elderly with DM in this country. In this current study, we explored the relationships between personality traits and QOL in elderly patients with DM in Malaysia, with the aim of contributing to the optimisation of disease management in this specific population.

\section{MATERIALS AND METHODS}

\section{Study Design and Participants}

This was a cross-sectional study conducted at the Universiti Kebangsaan Malaysia Medical Centre (UKMMC), a tertiary referral centre in Kuala Lumpur, Malaysia. Approval was obtained from the Research Ethics Committee of the Faculty of Medicine, Universiti Kebangsaan Malaysia (FF2019-342). Patients who attended outpatient clinics at UKMMC and who were interested in participating were provided with detailed explanations of the study. Participants were screened for inclusion criteria, including (i) being 60 years and above, and (ii) having a confirmed diagnosis of type 1 or type 2 DM. The diagnosis of DM was confirmed with medical records. Patients with conditions that impaired their mental capacity to give informed consent, for instance dementia and psychosis, were excluded from the study. All study participants gave their written informed consent before joining the study.

\section{Measuring Tools}

Study participants were asked to fill in a series of six self-administered questionnaires. The first two questionnaires asked details about demographics, social support, medical history, family history, history of diabetes, perceived management of diabetes, and medication adherence. Relevant recent laboratory results, including glycated haemoglobin (HbA1c) level, cholesterol levels, creatinine level, and albumin level were retrieved from the hospital's electronic databases. Another four validated instruments were used to evaluate personality traits, QOL, anxiety symptoms, and depressive symptoms.

The Big Five Inventory (BFI) was used to evaluate personality traits. The BFI is a 44-item, self-rated tool used to assess personality based on the five-factor model of personality i.e. openness to experience (10 items), conscientiousness (9 items), extraversion (8 items), agreeableness (9 items), and neuroticism (8 items) (John et al. 1991). The Malay version BFI showed good internal consistency as well as good convergent and discriminant validity (Muhammad et al. 2018). 
The World Health Organization Quality of Life-BREF (WHOQOLBREF) questionnaire is an abbreviated 26-item scale that was used to assess the domains of QOL (WHOQOL Group 1998b). The physical health domain of the WHOQOL-BREF questionnaire explores facets of QOL such as energy, mobility, pain and discomfort, sleep, capacity to work and to perform activities of daily living. The psychological health domain assesses body image, negative and positive feelings, self-esteem, spirituality and religiosity, and cognition. The social relationships domain explores aspects such as personal relationships, social support, and sexual activity. Finally, the environment domain enquires about financial resources, physical safety, home environment, participation in leisure activities, and transportation. A higher score indicates a better QOL in a particular domain. The Malay version of the WHOQOL-BREF has demonstrated good discriminant validity, construct validity, internal consistency, and test-retest reliability (Hasanah et al. 2003).

To control for the effects of mental illness on QOL, anxiety and depression were screened for using the 7-item Generalized Anxiety Disorder scale (GAD-7) and the Beck Depression Inventory-II (BDI-II), respectively. The GAD-7 is a self-reported questionnaire that has been found to have good reliability and validity in the screening of GAD (Spitzer et al. 2006). The Malay version of the GAD-7 was found to be valid and reliable in case-finding for anxiety with good sensitivity of $76 \%$ (95\% Cl $61-87 \%)$, a specificity of
94\% (88-97\%) (Sidik et al. 2012). The BDI-II is a self-reported questionnaire commonly used to screen for and assess the severity of depression. It is comprised of items that are related to depressive symptoms, such as hopelessness, guilt, and physical symptoms of fatigue (Beck et al. 1996). The Malay version of the instrument has been shown to have good validity and high level of internal consistency (Cronbach's $\alpha=0.71$ to 0.91 ) (Mukhtar \& Oei 2008).

\section{Data Analysis}

Statistical analysis was carried out using the Statistical Package for Social Science (SPSS) version 20 (IBM Corp., Armonk, NY, USA). Descriptive statistics were computed in which categorical variables were reported in frequency and percentages, and continuous variables reported in median and interquartile range (IQR). The continuous variables were not normally distributed as demonstrated by the Kolmogorov-Smirnov test $(\mathrm{P}<$ 0.05). Correlations between continuous variables in the WHOQOL-BREF domain scores were calculated using the Spearman's correlation coefficients. The comparisons of the WHOQOLBREF domain scores across categorical variables were evaluated with the Mann-Whitney $U$ test and the KruskalWallis test. Variables with significant differences from bivariate analyses were then entered into stepwise backward linear regression models for each QOL domains.

All the multiple linear regression models were checked to confirm 
that the assumptions for multiple linear regression were satisfied i.e., (i) Scatterplot of the standardised residuals with the standardised predicted values were plotted with a Loess curve fitted through it. The relationship of standardised predicted to residuals was roughly linear around zero, indicating that the relationship between the dependent variable and the predictors was linear; (ii) Additionally, the variance around zero was scattered uniformly and randomly, thus showing homoscedasticity; (iii) In the Q-Q plot for standardised residuals, the points clustered around the diagonal line, indicating normal distribution for residuals; (iv) The variance inflation factor of all the independent variables were less than 10 and tolerance scores of all the independent variables in all the models were above 0.1, suggesting no multicollinearity between the independent variables; (v) Multivariate outliers were checked with the Mahalanobis Distance for the variables. Cases with the probability of $<0.001$ for the chi-square distribution of Mahalanobis Distance were flagged as multivariate outliers and excluded from the regression analyses. Statistical significance for all analyses was set to $\mathrm{P}<0.05$.

\section{RESULTS}

\section{Characteristics of Study Participants}

A total of 170 study participants were included in the analyses. The median age of participants was 69 years (IQR: 65.0-73.0) and a slight majority of participants were male
(52.1\%). In terms of ethnicity, $62.4 \%$ of participants were Malay, 19.4\% were Chinese, and $16.5 \%$ of participants were Indian. Nearly two-thirds of participants were Muslim (64.1\%), while the remainder of participants practised Buddhism (14.1\%), Hinduism (11.8\%), or Christianity (7.6\%). In addition, most participants were married $(78.2 \%)$, retired $(61.2 \%)$, and reported a household income of less than <RM 3,000 (65.3\%).

Most participants reported having good social support $(81.8 \%)$ and strong religiosity $(80.0 \%)$. In terms of activity levels, roughly three-quarters of participants reported having an active lifestyle (75.3\%) and a healthy diet $(74.7 \%)$. Finally, most participants were non-smokers $(73.5 \%)$, did not consume alcohol (91.8\%), and had no history of recreational drug use (97.6\%).

Regarding clinical characteristics, the median duration of diabetes was 15.0 years (IQR: 10.0-20.5). Approximately two thirds (65.3\%) of participants had poor glycaemic control, defined as HbA1c levels $>7.0 \%$, and nearly half of the participants were on insulin therapy (40.6\%). Despite these findings, threequarters of participants perceived their diabetes to be well-managed (75.9\%). In terms of laboratory findings, the median HbA1c was 7.3\% (IQR 6.48.8), the median LDL cholesterol was 2.3 (IQR 1.9-3.0), and the median HDL cholesterol was 1.2 (IQR 1.0-1.4).

The median scores for the QOL domains were 14.0 for physical health (IQR: 12.6-15.4), 15.3 for psychological health (IQR: 13.9-16.7), 16.0 for social relationships (IQR: 13.3-16.0), and 15.0 for the environment (IQR: 13.66-16.00). 
Table 1: Sociodemographic and clinical characteristics of the study sample

\begin{tabular}{|c|c|c|}
\hline Variable & $\mathbf{n}$ & $\%$ \\
\hline \multicolumn{3}{|l|}{ Gender } \\
\hline Male & 87 & 51.2 \\
\hline Female & 83 & 48.8 \\
\hline \multicolumn{3}{|l|}{ Race } \\
\hline Malay & 106 & 62.4 \\
\hline Chinese & 33 & 19.4 \\
\hline Indian & 28 & 16.5 \\
\hline Other & 3 & 1.8 \\
\hline \multicolumn{3}{|l|}{ Marital status } \\
\hline Married & 133 & 78.2 \\
\hline Single & 4 & 2.4 \\
\hline Divorced/separated & 5 & 2.9 \\
\hline Widowed & 28 & 16.5 \\
\hline \multicolumn{3}{|l|}{ Education } \\
\hline None & 6 & 3.5 \\
\hline Primary school & 33 & 19.4 \\
\hline Secondary school & 78 & 45.9 \\
\hline Diploma & 21 & 12.4 \\
\hline Undergraduate degree & 13 & 7.6 \\
\hline Postgraduate degree & 17 & 10.0 \\
\hline Missing & 2 & 1.2 \\
\hline \multicolumn{3}{|l|}{ Employment } \\
\hline Employed & 51 & 30.0 \\
\hline Unemployed & 13 & 7.6 \\
\hline Retired & 104 & 61.2 \\
\hline Missing & 2 & 1.2 \\
\hline \multicolumn{3}{|l|}{ Household income } \\
\hline Employed & 111 & 65.3 \\
\hline Unemployed & 24 & 14.1 \\
\hline Retired & 24 & 14.1 \\
\hline Missing & 11 & 6.5 \\
\hline \multicolumn{3}{|l|}{ Religion } \\
\hline Islam & 109 & 64.1 \\
\hline Buddhism & 24 & 14.1 \\
\hline Hinduism & 20 & 11.8 \\
\hline Christianity & 13 & 7.6 \\
\hline Other & 3 & 1.8 \\
\hline Missing & 1 & 0.6 \\
\hline \multicolumn{3}{|l|}{ Practice of religion } \\
\hline Strongly disagree & 9 & 5.3 \\
\hline Disagree & 3 & 1.8 \\
\hline Neutral & 22 & 12.9 \\
\hline Agree & 69 & 40.6 \\
\hline Strongly agree & 67 & 39.4 \\
\hline \multicolumn{3}{|l|}{ Strong social support } \\
\hline Strongly disagree & 1 & 0.6 \\
\hline Disagree & 3 & 1.8 \\
\hline Neutral & 27 & 15.9 \\
\hline Agree & 97 & 57.1 \\
\hline Strongly agree & 42 & 24.7 \\
\hline
\end{tabular}




\begin{tabular}{|c|c|c|}
\hline Variable & $\mathbf{n}$ & $\%$ \\
\hline \multicolumn{3}{|l|}{ Active lifestyle } \\
\hline Strongly disagree & 2 & 1.2 \\
\hline Disagree & 3 & 1.8 \\
\hline Neutral & 37 & 21.8 \\
\hline Agree & 100 & 58.8 \\
\hline Strongly agree & 28 & 16.5 \\
\hline \multicolumn{3}{|c|}{ Healthy eating pattern } \\
\hline Strongly disagree & 2 & 1.2 \\
\hline Disagree & 5 & 2.9 \\
\hline Neutral & 35 & 20.6 \\
\hline Agree & 101 & 59.4 \\
\hline Strongly agree & 26 & 15.3 \\
\hline Missing & 1 & 0.6 \\
\hline \multicolumn{3}{|l|}{ Smoking } \\
\hline Never & 125 & 73.5 \\
\hline Ex-smoker & 39 & 22.9 \\
\hline Current smoker & 6 & 3.5 \\
\hline \multicolumn{3}{|l|}{ Alcohol use } \\
\hline Yes & 12 & 7.1 \\
\hline No & 156 & 91.8 \\
\hline Missing & 2 & 1.2 \\
\hline \multicolumn{3}{|l|}{ Recreational drug use } \\
\hline Yes & 3 & 1.8 \\
\hline No & 166 & 97.6 \\
\hline Missing & 1 & 0.6 \\
\hline \multicolumn{3}{|l|}{ Insulin therapy } \\
\hline Yes & 69 & 40.6 \\
\hline No & 75 & 44.1 \\
\hline Missing & 26 & 15.3 \\
\hline \multicolumn{3}{|c|}{ 'I am able to manage my diabetes well' } \\
\hline Strongly disagree & 3 & 1.8 \\
\hline Disagree & 8 & 4.7 \\
\hline Neutral & 29 & 17.1 \\
\hline Agree & 68 & 40.0 \\
\hline Strongly agree & 61 & 35.9 \\
\hline Missing & 1 & 0.6 \\
\hline \multicolumn{3}{|l|}{ Diabetic control } \\
\hline Good & 59 & 34.7 \\
\hline Poor & 111 & 65.3 \\
\hline \multicolumn{3}{|l|}{ Obesity } \\
\hline $\mathrm{BMI}<25$ & 43 & 25.3 \\
\hline BMI 25-30 & 46 & 27.1 \\
\hline $\mathrm{BMI}>30$ & 35 & 20.6 \\
\hline Missing & 46 & 27.1 \\
\hline \multicolumn{3}{|l|}{ Hypertension } \\
\hline Yes & 143 & 84.1 \\
\hline No & 27 & 15.9 \\
\hline \multicolumn{3}{|l|}{ Dyslipidaemia } \\
\hline Yes & 95 & 55.9 \\
\hline No & 75 & 44.1 \\
\hline
\end{tabular}




\begin{tabular}{lcc}
\hline Variable & $\mathbf{n}$ & $\mathbf{\%}$ \\
\hline Ischaemic heart disease & & \\
Yes & 59 & 34.7 \\
No & 111 & 65.3 \\
Stroke & & \\
Yes & 18 & 10.6 \\
No & 152 & 89.4 \\
Renal disease & & \\
Yes & 27 & 15.9 \\
No & 143 & 84.1 \\
\hline
\end{tabular}

A complete list of sociodemographic and clinical characteristics collected can be seen in Table 1 and a summary of laboratory findings and outcome measure scores can be found in Table 2.

\section{Findings from Bivariate Analysis}

The results of bivariate analysis are shown in Table 3 and Table 4. For the physical health domain, the score was positively associated with $\mathrm{HDL}$ cholesterol level, albumin level, BFI extraversion, agreeableness, and conscientiousness scores, while negatively associated with age,

Table 2: Laboratory results, BMI, GAD-7, BDI, BFI and WHOQOL-BREF scores of the study sample

\begin{tabular}{lcc}
\hline Variable & Median & IQR \\
\hline HbA1c (\%) & 7.3 & $6.4-8.8$ \\
LDL & 2.3 & $1.9-3.0$ \\
HDL & 1.2 & $1.0-1.4$ \\
Creatinine & 104.1 & $76.0-183.6$ \\
Albumin & 39.0 & $36.0-41.0$ \\
BMI & 26.9 & $23.7-30.1$ \\
GAD-7 & 0.0 & $0.0-3.0$ \\
BDI-II & 4.0 & $2.0-7.0$ \\
BFI & & \\
Extraversion & 3.4 & $3.0-3.8$ \\
Agreeableness & 3.9 & $3.6-4.1$ \\
Conscientiousness & 3.7 & $3.4-4.0$ \\
Neuroticism & 2.5 & $2.0-2.8$ \\
Openness & 3.2 & $3.0-3.5$ \\
WHOQOL-BREF & & \\
Physical health & 14.0 & $12.6-15.4$ \\
Psychological & 15.3 & $13.9-16.7$ \\
Social relationships & 16.0 & $13.3-16.0$ \\
Environment & 15.0 & $13.7-16.0$ \\
\hline
\end{tabular}


Table 3: Relationship between domains of WHOQOL-BREF and continuous variables in the study

\begin{tabular}{|c|c|c|c|c|}
\hline Variable & Physical health & Psychological & $\begin{array}{l}\text { Social } \\
\text { relationships }\end{array}$ & Environment \\
\hline \multicolumn{5}{|l|}{ Age } \\
\hline$n$ & 170 & 170 & 170 & 170 \\
\hline Spearman's r & $-0.188^{*}$ & -0.08 & -0.06 & $-0.171^{*}$ \\
\hline \multicolumn{5}{|l|}{ Body mass index } \\
\hline & 123 & 123 & 123 & 123 \\
\hline Spearman's $r$ & 0.06 & 0.10 & 0.14 & 0.15 \\
\hline \multicolumn{5}{|l|}{ Duration of DM } \\
\hline & 157 & 157 & 157 & 157 \\
\hline Spearman's $r$ & -0.14 & $-0.212^{* *}$ & -0.15 & $-0.195^{*}$ \\
\hline \multicolumn{5}{|l|}{ HbA1c } \\
\hline & 153 & 153 & 153 & 153 \\
\hline Spearman's $r$ & 0.00 & -0.10 & -0.06 & -0.11 \\
\hline \multicolumn{5}{|l|}{ LDL cholesterol } \\
\hline & 151 & 151 & 151 & 151 \\
\hline Spearman's $r$ & -0.07 & 0.09 & 0.05 & 0.05 \\
\hline \multicolumn{5}{|l|}{ HDL cholesterol } \\
\hline & 153 & 153 & 153 & 153 \\
\hline Spearman's r & $0.209^{* *}$ & $0.225^{* *}$ & $0.196^{*}$ & $0.235^{* *}$ \\
\hline \multicolumn{5}{|l|}{ Creatinine } \\
\hline & 158 & 158 & 158 & 158 \\
\hline Spearman's r & $-0.212^{* *}$ & -0.05 & -0.04 & $-0.174^{*}$ \\
\hline \multicolumn{5}{|l|}{ Albumin } \\
\hline & 155 & 155 & 155 & 155 \\
\hline Spearman's $r$ & $0.301^{* *}$ & 0.15 & 0.13 & 0.16 \\
\hline \multicolumn{5}{|l|}{ GAD-7 score } \\
\hline & 170 & 170 & 170 & 170 \\
\hline Spearman's r & $-0.301^{* *}$ & $-0.405^{* *}$ & $-0.297^{* *}$ & $-0.319 * *$ \\
\hline \multicolumn{5}{|l|}{ BDI-II score } \\
\hline & 170 & 170 & 170 & 170 \\
\hline Spearman's $r$ & $-0.482^{* *}$ & $-0.411^{* *}$ & $-0.347^{* *}$ & $-0.333^{* *}$ \\
\hline \multicolumn{5}{|l|}{ BFI extraversion } \\
\hline & 170 & 170 & 170 & 170 \\
\hline Spearman's $r$ & $0.270^{* *}$ & $0.440^{* *}$ & $0.346^{* *}$ & $0.444^{* *}$ \\
\hline \multicolumn{5}{|l|}{ BFI agreeableness } \\
\hline & 170 & 170 & 170 & 170 \\
\hline Spearman's $r$ & $0.231^{* *}$ & $0.432 * *$ & $0.451^{* *}$ & $0.527^{* *}$ \\
\hline \multicolumn{5}{|c|}{ BFI conscientiousness } \\
\hline$n$ & 170 & 170 & 170 & 170 \\
\hline Spearman's $r$ & $0.367^{* *}$ & $0.420^{* *}$ & $0.411^{* *}$ & $0.482^{* *}$ \\
\hline \multicolumn{5}{|l|}{ BFI neuroticism } \\
\hline$n$ & 170 & 170 & 170 & 170 \\
\hline Spearman's $r$ & $-0.391^{* *}$ & $-0.582^{* *}$ & $-0.453^{* *}$ & $-0.530^{* *}$ \\
\hline \multicolumn{5}{|l|}{ BFI openness } \\
\hline$n$ & 170 & 170 & 170 & 170 \\
\hline Spearman's r & 0.14 & 0.12 & 0.11 & $0.210^{* *}$ \\
\hline$* \mathrm{P}<0.05 ; * * \mathrm{P}<0.0$ & & & & \\
\hline
\end{tabular}


Table 4: Relationship between domains of WHOQOL-BREF and categorical variables in the study

\begin{tabular}{|c|c|c|c|c|c|c|c|c|}
\hline \multirow[t]{2}{*}{ Variable } & \multicolumn{2}{|c|}{ Physical health } & \multicolumn{2}{|c|}{ Psychological } & \multicolumn{2}{|c|}{$\begin{array}{l}\text { Social } \\
\text { relationships }\end{array}$} & \multicolumn{2}{|c|}{ Environment } \\
\hline & Median & IQR & Median & IQR & Median & IQR & Median & IQR \\
\hline \multicolumn{9}{|l|}{ Gender $^{\mathrm{a}}$} \\
\hline Male & 14.29 & 3.43 & 15.33 & 3.07 & 16.00 & 2.67 & 15.00 & 2.29 \\
\hline Female & 13.71 & 3.43 & 15.33 & 2.67 & 16.00 & 2.67 & 15.00 & 2.50 \\
\hline $\mathrm{P}$ & 0.368 & & 0.931 & & 0.389 & & 0.893 & \\
\hline \multicolumn{9}{|l|}{ Race $^{b}$} \\
\hline Malay & 14.29 & 3.43 & 15.33 & 2.67 & 16.00 & 2.67 & 15.00 & 1.63 \\
\hline Chinese & 13.71 & 4.00 & 15.33 & 4.00 & 14.67 & 2.67 & 14.50 & 3.00 \\
\hline Indian & 13.71 & 2.29 & 14.67 & 2.49 & 15.34 & 4.00 & 15.00 & 3.88 \\
\hline Others & 13.71 & 5.14 & 16.00 & 0.00 & 16.00 & 0.00 & 13.50 & 0.00 \\
\hline $\mathrm{P}$ & 0.715 & & 0.178 & & 0.263 & & 0.312 & \\
\hline \multicolumn{9}{|l|}{ Marital status ${ }^{\mathrm{b}}$} \\
\hline Married & 14.29 & 3.43 & 15.33 & 3.21 & 16.00 & 2.67 & 15.00 & 3.00 \\
\hline Single & 13.15 & 5.43 & 15.60 & 4.10 & 16.00 & 7.33 & 15.00 & 3.63 \\
\hline $\begin{array}{l}\text { Divorced/ } \\
\text { separated }\end{array}$ & 14.29 & 7.58 & 16.34 & 6.66 & 14.00 & 5.99 & 15.50 & 2.50 \\
\hline Widowed & 13.43 & 2.72 & 15.33 & 3.01 & 16.00 & 2.50 & 14.75 & 1.50 \\
\hline $\mathrm{P}$ & 0.455 & & 0.994 & & 0.645 & & 0.760 & \\
\hline \multicolumn{9}{|l|}{ Education $^{b}$} \\
\hline None & 16.00 & 7.72 & 17.33 & 7.00 & 16.00 & 12.00 & 16.00 & 8.75 \\
\hline Primary school & 13.43 & 2.86 & 15.00 & 3.00 & 16.00 & 4.00 & 14.75 & 2.63 \\
\hline Secondary school & 13.71 & 2.86 & 15.20 & 2.67 & 16.00 & 4.00 & 14.50 & 2.00 \\
\hline Diploma & 14.58 & 3.14 & 15.33 & 2.34 & 16.00 & 2.67 & 15.00 & 2.88 \\
\hline $\begin{array}{l}\text { Undergraduate } \\
\text { degree }\end{array}$ & 15.03 & 2.29 & 15.67 & 3.36 & 16.00 & 2.33 & 16.00 & 3.63 \\
\hline Postgraduate degree & 14.86 & 4.57 & 17.33 & 2.67 & 16.00 & 4.00 & 17.50 & 2.50 \\
\hline $\mathrm{P}$ & 0.134 & & 0.056 & & 0.725 & & $0.002 * *$ & \\
\hline \multicolumn{9}{|l|}{ Employment $^{\mathrm{b}}$} \\
\hline Employed & 13.33 & 2.86 & 14.00 & 4.00 & 16.00 & 4.00 & 14.00 & 3.00 \\
\hline Unemployed & 13.71 & 2.86 & 15.33 & 3.33 & 14.67 & 4.00 & 16.50 & 5.00 \\
\hline Retired & 14.86 & 2.86 & 15.33 & 2.67 & 16.00 & 2.67 & 15.00 & 1.50 \\
\hline $\mathrm{P}$ & $0.016^{*}$ & & $0.030^{*}$ & & 0.120 & & $0.003^{* *}$ & \\
\hline \multicolumn{9}{|l|}{ Household income $^{b}$} \\
\hline$<\mathrm{RM} 3,000$ & 13.71 & 2.86 & 15.27 & 2.84 & 16.00 & 2.67 & 14.50 & 2.50 \\
\hline RM3,000-6,000 & 13.71 & 2.86 & 15.33 & 2.67 & 16.00 & 5.33 & 15.50 & 4.00 \\
\hline$>\mathrm{RM} 6,000$ & 15.15 & 4.00 & 16.00 & 2.00 & 16.00 & 2.67 & 16.50 & 3.25 \\
\hline $\mathrm{P}$ & 0.240 & & $0.019^{*}$ & & 0.712 & & $<0.001^{* *}$ & \\
\hline \multicolumn{9}{|l|}{ Religion $^{b}$} \\
\hline Islam & 14.29 & 3.43 & 15.33 & 2.67 & 16.00 & 2.67 & 15.00 & 2.00 \\
\hline Buddhism & 13.71 & 4.29 & 16.00 & 4.00 & 14.67 & 4.00 & 14.50 & 4.00 \\
\hline Hinduism & 13.14 & 3.00 & 14.67 & 2.73 & 15.34 & 4.33 & 15.00 & 5.13 \\
\hline Christianity & 14.29 & 2.86 & 14.00 & 3.00 & 14.67 & 3.34 & 15.50 & 3.75 \\
\hline Other & 13.14 & 0.00 & 12.00 & 0.00 & 14.67 & 0.00 & 12.50 & 0.00 \\
\hline $\mathrm{P}$ & 0.245 & & $0.025^{*}$ & & 0.399 & & 0.273 & \\
\hline \multicolumn{9}{|l|}{ Practice of religion ${ }^{b}$} \\
\hline Strongly disagree & 14.00 & 6.72 & 13.67 & 8.17 & 14.00 & 6.33 & 14.75 & 4.63 \\
\hline Disagree & 12.00 & 0.00 & 14.67 & 0.00 & 14.67 & 0.00 & 13.00 & 0.00 \\
\hline Neutral & 13.72 & 4.43 & 15.33 & 4.66 & 15.34 & 4.00 & 14.50 & 4.75 \\
\hline Agree & 14.29 & 2.57 & 14.40 & 2.67 & 16.00 & 2.67 & 14.50 & 2.00 \\
\hline Strongly agree & 14.29 & 3.14 & 16.00 & 2.00 & 16.00 & 2.34 & 15.50 & 3.00 \\
\hline $\mathrm{P}$ & 0.411 & & $0.001^{* *}$ & & 0.306 & & $<0.001^{* *}$ & \\
\hline
\end{tabular}




\begin{tabular}{|c|c|c|c|c|c|c|c|c|}
\hline \multirow[t]{2}{*}{ Variable } & \multicolumn{2}{|c|}{ Physical health } & \multicolumn{2}{|c|}{ Psychological } & \multicolumn{2}{|c|}{$\begin{array}{l}\text { Social } \\
\text { relationships }\end{array}$} & \multicolumn{2}{|c|}{ Environment } \\
\hline & Median & IQR & Median & IQR & Median & IQR & Median & IQR \\
\hline \multicolumn{9}{|l|}{ Social support ${ }^{b}$} \\
\hline Disagree & 10.29 & 0.00 & 11.33 & 0.00 & 10.67 & 0.00 & 10.50 & 0.00 \\
\hline Less agree & 13.71 & 0.00 & 14.67 & 0.00 & 14.67 & 0.00 & 14.50 & 0.00 \\
\hline Neutral & 12.57 & 3.43 & 13.33 & 3.33 & 12.00 & 4.00 & 14.00 & 2.35 \\
\hline Agree & 14.29 & 2.86 & 15.33 & 2.67 & 16.00 & 2.00 & 14.50 & 2.50 \\
\hline Very agree & 14.29 & 2.86 & 16.00 & 2.67 & 16.00 & 5.34 & 17.00 & 3.50 \\
\hline $\mathrm{P}$ & $0.007^{* *}$ & & $<0.001^{* *}$ & & $<0.001^{* *}$ & & $<0.001^{* *}$ & \\
\hline \multicolumn{9}{|l|}{ Active lifestyle $^{b}$} \\
\hline Strongly disagree & 10.86 & 0.00 & 10.67 & 0.00 & 9.34 & 0.00 & 11.25 & 0.00 \\
\hline Disagree & 12.00 & 0.00 & 13.33 & 0.00 & 16.00 & 0.00 & 14.00 & 0.00 \\
\hline Neutral & 12.86 & 2.86 & 13.34 & 3.33 & 14.34 & 4.00 & 13.86 & 3.50 \\
\hline Agree & 14.25 & 3.09 & 15.33 & 2.54 & 16.00 & 2.67 & 15.00 & 2.00 \\
\hline Strongly agree & 14.86 & 3.43 & 17.33 & 2.67 & 16.00 & 5.00 & 17.00 & 3.50 \\
\hline $\mathrm{P}$ & $0.001^{* *}$ & & $0.001^{* *}$ & & $0.006^{* *}$ & & $<0.001^{* *}$ & \\
\hline \multicolumn{9}{|c|}{ Healthy eating pattern ${ }^{b}$} \\
\hline Strongly disagree & 9.43 & 0.00 & 10.40 & 0.00 & 8.00 & 0.00 & 11.00 & 0.00 \\
\hline Disagree & 12.29 & 3.14 & 12.67 & 3.33 & 12.00 & 4.66 & 13.50 & 3.25 \\
\hline Neutral & 13.71 & 3.43 & 14.67 & 3.34 & 14.67 & 4.00 & 14.50 & 3.00 \\
\hline Agree & 13.71 & 2.86 & 15.33 & 2.20 & 16.00 & 2.67 & 15.00 & 2.00 \\
\hline Strongly agree & 14.86 & 2.85 & 16.67 & 2.67 & 16.00 & 6.00 & 17.50 & 4.00 \\
\hline $\mathrm{P}$ & $0.009 * *$ & & $0.003^{* *}$ & & $0.005^{* *}$ & & $<0.001^{* *}$ & \\
\hline \multicolumn{9}{|l|}{ Smoking ${ }^{b}$} \\
\hline Never & 14.00 & 3.14 & 15.33 & 2.77 & 16.00 & 2.67 & 15.00 & 2.50 \\
\hline Ex-smoker & 13.71 & 3.00 & 15.33 & 4.00 & 14.67 & 4.00 & 14.50 & 2.50 \\
\hline Current smoker & 14.29 & 3.05 & 15.33 & 2.00 & 16.00 & 1.33 & 14.00 & 3.75 \\
\hline $\mathrm{P}$ & 0.867 & & 0.505 & & 0.524 & & 0.392 & \\
\hline \multicolumn{9}{|l|}{ Alcohol use $\mathrm{a}^{\mathrm{a}}$} \\
\hline Yes & 14.29 & 2.43 & 15.33 & 2.33 & 16.00 & 1.00 & 15.50 & 3.13 \\
\hline No & 13.71 & 3.14 & 15.33 & 3.34 & 16.00 & 2.67 & 15.00 & 2.39 \\
\hline $\mathrm{P}$ & 0.510 & & 0.605 & & 0.177 & & 0.312 & \\
\hline \multicolumn{9}{|c|}{ Recreational drug use } \\
\hline Yes & 13.71 & 0.00 & 12.00 & 0.00 & 14.67 & 0.00 & 13.50 & 0.00 \\
\hline No & 14.29 & 3.43 & 15.33 & 2.77 & 16.00 & 2.67 & 15.00 & 2.57 \\
\hline $\mathrm{P}$ & 0.595 & & 0.369 & & 0.660 & & 0.373 & \\
\hline \multicolumn{9}{|l|}{ Insulin therapy ${ }^{a}$} \\
\hline Yes & 14.00 & 2.72 & 15.33 & 3.34 & 16.00 & 2.67 & 15.00 & 3.50 \\
\hline No & 14.86 & 2.86 & 16.00 & 2.00 & 16.00 & 2.67 & 15.00 & 2.50 \\
\hline $\mathrm{P}$ & 0.200 & & 0.138 & & 0.806 & & 0.366 & \\
\hline \multicolumn{9}{|l|}{$\begin{array}{l}\text { 'I am able to manage } \\
\text { my diabetes well’b }\end{array}$} \\
\hline Strongly disagree & 13.71 & 0.00 & 12.00 & 0.00 & 13.33 & 0.00 & 13.50 & 0.00 \\
\hline Disagree & 12.00 & 0.00 & 14.67 & 0.00 & 14.67 & 0.00 & 12.50 & 0.00 \\
\hline Neutral & 12.57 & 3.43 & 13.33 & 4.00 & 13.33 & 4.00 & 13.50 & 2.50 \\
\hline Agree & 14.58 & 2.41 & 15.33 & 1.60 & 16.00 & 2.67 & 15.00 & 2.13 \\
\hline Strongly agree & 14.86 & 2.86 & 15.33 & 3.33 & 16.00 & 2.67 & 15.43 & 4.00 \\
\hline $\mathrm{P}$ & $<0.001^{* *}$ & & $<0.001^{* *}$ & & $0.003^{* *}$ & & $<0.001^{* *}$ & \\
\hline \multicolumn{9}{|l|}{ Diabetic controla } \\
\hline Good & 14.29 & 2.86 & 15.33 & 2.13 & 16.00 & 2.00 & 15.43 & 2.50 \\
\hline Poor & 14.29 & 3.29 & 15.33 & 3.34 & 16.00 & 2.67 & 14.75 & 3.38 \\
\hline $\mathrm{P}$ & 0.364 & & 0.062 & & 0.235 & & 0.127 & \\
\hline
\end{tabular}




\begin{tabular}{|c|c|c|c|c|c|c|c|c|}
\hline \multirow[t]{2}{*}{ Variable } & \multicolumn{2}{|c|}{ Physical health } & \multicolumn{2}{|c|}{ Psychological } & \multicolumn{2}{|c|}{$\begin{array}{c}\text { Social } \\
\text { relationships }\end{array}$} & \multicolumn{2}{|c|}{ Environment } \\
\hline & Median & IQR & Median & IQR & Median & IQR & Median & IQR \\
\hline Obesity ${ }^{b}$ & 14.58 & 2.86 & 15.33 & 2.50 & 16.00 & 2.67 & 15.00 & 2.82 \\
\hline BMI <25 & 13.71 & 3.43 & 15.33 & 3.34 & 16.00 & 4.00 & 14.50 & 3.50 \\
\hline BMI 25-30 & 14.29 & 3.26 & 15.33 & 3.83 & 16.00 & 3.67 & 15.50 & 4.00 \\
\hline $\mathrm{BMI}>30$ & 0.657 & & 0.563 & & 0.584 & & 0.119 & \\
\hline $\mathrm{P}$ & & & & & & & & \\
\hline \multicolumn{9}{|l|}{ Hypertension ${ }^{\mathrm{a}}$} \\
\hline Yes & 14.00 & 2.86 & 15.33 & 3.34 & 16.00 & 2.67 & 15.00 & 2.13 \\
\hline No & 14.86 & 4.00 & 16.00 & 2.66 & 16.00 & 4.34 & 15.50 & 4.25 \\
\hline $\mathrm{P}$ & $0.009 * *$ & & $0.041^{*}$ & & 0.052 & & 0.072 & \\
\hline \multicolumn{9}{|c|}{ Dyslipidaemia $^{a}$} \\
\hline Yes & 14.29 & 2.86 & 15.33 & 2.67 & 16.00 & 2.67 & 15.00 & 2.00 \\
\hline No & 14.00 & 2.86 & 15.33 & 2.67 & 14.67 & 2.67 & 15.00 & 3.38 \\
\hline $\mathrm{P}$ & 0.301 & & 0.972 & & 0.950 & & 0.743 & \\
\hline \multicolumn{9}{|c|}{$\begin{array}{l}\text { Ischaemic heart } \\
\text { disease }^{\text {a }}\end{array}$} \\
\hline Yes & 13.71 & 2.43 & 15.33 & 4.00 & 15.34 & 4.00 & 15.00 & 2.75 \\
\hline No & 14.29 & 2.52 & 15.33 & 2.67 & 16.00 & 2.67 & 15.00 & 2.75 \\
\hline $\mathrm{P}$ & $0.026^{*}$ & & 0.541 & & 0.456 & & 0.077 & \\
\hline \multicolumn{9}{|l|}{ Stroke ${ }^{a}$} \\
\hline Yes & 13.14 & 2.86 & 15.33 & 4.00 & 14.67 & 4.00 & 14.50 & 1.50 \\
\hline No & 14.29 & 2.86 & 15.33 & 2.67 & 16.00 & 2.67 & 15.00 & 3.00 \\
\hline $\mathrm{P}$ & $0.047^{*}$ & & 0.055 & & $0.044^{*}$ & & 0.103 & \\
\hline \multicolumn{9}{|l|}{ Renal disease } \\
\hline Yes & 13.14 & 2.86 & 15.33 & 4.33 & 14.67 & 4.00 & 14.50 & 3.63 \\
\hline No & 14.29 & 3.09 & 15.33 & 2.67 & 16.00 & 2.67 & 15.00 & 3.00 \\
\hline $\mathrm{P}$ & 0.149 & & 0.884 & & 0.714 & & 0.427 & \\
\hline
\end{tabular}

aMann-Whitney $U$ test; ${ }^{\text {b}}$ Kruskal-Wallis test

* $\mathrm{P}<0.05$; ** $\mathrm{P}<0.01$; *** $\mathrm{P}<0.001$

creatinine level, GAD-7 score, BDIII score, and BFI neuroticism scores. Retired employment status, greater affirmation of social support, practice of active lifestyle, healthy eating pattern, and perceived self-efficacy in diabetic management were associated with higher score. Meanwhile, hypertension, ischaemic heart disease, and stroke were associated with lower score in the physical health domain.

For the psychological domain, the score was positively associated with HDL cholesterol level, BFI extraversion, agreeableness, and conscientiousness scores, but negatively associated with duration of DM, GAD-7 score, BDIII score, and BFI neuroticism score. Higher household income, Buddhism as religion, greater affirmation of practice of religion, social support, practice of active lifestyle, healthy eating pattern, and perceived selfefficacy in diabetic management were associated with higher score in the psychological domain, whereas employed status, and hypertension were associated with lower score.

As for social relationships, $\mathrm{HDL}$ cholesterol level, BFI extraversion, agreeableness, and conscientiousness scores showed positive associations, 
while GAD-7 score, BDI-II score, and $\mathrm{BFI}$ neuroticism score showed negative association with the domain score. Stronger affirmation of social support, practice of active lifestyle, healthy eating pattern, and perceived selfefficacy in diabetic management were associated with higher score for social relationships domain, but stroke was associated with lower socre.

Finally, significant variables from bivariate analysis that had positive association with the environment domain score were HDL cholesterol level, BFI extraversion, agreeableness, conscientiousness, and openness scores, while age, duration of DM, creatinine level, GAD-7 score, BDI-II score, and BFI neuroticism score had negative association with the domain score. Education at postgraduate level, being unemployed, high household income, higher affirmation of the practice of religion, social support, practice of active lifestyle, healthy eating pattern, and perceived selfefficacy in diabetic management were also associated with higher score for the environment domain.

\section{Stepwise Linear Regression Analysis}

Regression analysis showed that, after controlling for other factors, higher conscientiousness scores $\quad(\beta=0.156$; $\mathrm{P}=0.044)$ and lower neuroticism scores $(\beta=-0.176 ; P=0.028)$ on the $\mathrm{BFI}$ were significantly associated with a greater QOL in the physical health domain. Furthermore, higher BFI extraversion scores $(\beta=0.209 ; P=0.001)$ and higher BFI conscientiousness scores $(\beta=0.248$; $\mathrm{P}<0.001)$ were each associated with a greater psychological QOL. In addition, higher extraversion scores $(\beta=0.172$; $\mathrm{P}=0.012)$ and agreeableness scores $(\beta=0.286 ; \quad P<0.001)$ were associated with a better social relationships QOL. Finally, higher extraversion scores $(\beta=0.202 ; \quad P=0.002)$, higher agreeableness scores $\quad(\beta=0.327$; $P<0.001)$, and lower neuroticism scores $(\beta=-0.223 ; P=0.001)$ on the $B F I$ were associated with a better QOL in the environment domain (Table 5).

\section{DISCUSSION}

In this study, we evaluated the relationship between personality and QOL in elderly patients with DM. Not only is diabetes more prevalent among the elderly population due to its chronicity, but patients in this age group also face their own unique challenges in terms of managing and living with their diabetes illness. To the best of our knowledge, our study is the first to examine the relationship between personality and QOL, specifically in a sample of elderly patients with DM in Malaysia. In keeping with the current literature, we found there to be an association between personality traits and QOL. More specifically, we found an association between personality traits and each of the individual domains of the WHOQOL-BREF questionnaire-physical, psychological, social relationship, and environmental.

Studies have previously established an association between personality and QOL in the general diabetic population. In fact, in a large population sample of patients with type 2 DM, Imayama et al. (2011) found that personality was 
Table 5: Significant factors for each domain of WHOQOL-BREF in stepwise linear regression analyses

\begin{tabular}{|c|c|c|c|c|c|}
\hline Domain & Variable & $R^{2}$ & $B$ & $\beta$ & $P$ \\
\hline \multirow[t]{7}{*}{ Physical health } & Constant & & 14.065 & & \\
\hline & Age & 0.244 & -0.058 & -0.138 & 0.047 \\
\hline & Albumin & 0.300 & 0.093 & 0.179 & 0.010 \\
\hline & BDI-II score & 0.330 & -0.134 & -0.331 & $<0.001$ \\
\hline & BFI conscientiousness & 0.357 & 0.748 & 0.156 & 0.044 \\
\hline & BFI neuroticism & 0.375 & -0.703 & -0.176 & 0.029 \\
\hline & Fitness test: $F=17.040, P<0.001$ & & & & \\
\hline \multirow[t]{8}{*}{ Psychological } & Constant & & 5.860 & & \\
\hline & Practice of religion & 0.377 & 0.326 & 0.140 & 0.013 \\
\hline & Active lifestyle & 0.523 & 0.452 & 0.146 & 0.011 \\
\hline & Duration of DM & 0.558 & -0.043 & -0.186 & 0.001 \\
\hline & GAD-7 score & 0.592 & -0.255 & -0.446 & $<0.001$ \\
\hline & BFI extraversion & 0.615 & 0.912 & 0.209 & 0.001 \\
\hline & BFI conscientiousness & 0.634 & 1.149 & 0.248 & $<0.001$ \\
\hline & Fitness test: $F=36.324, P<0.001$ & & & & \\
\hline \multirow{6}{*}{$\begin{array}{l}\text { Social } \\
\text { relationships }\end{array}$} & Constant & & 1.887 & & \\
\hline & Healthy eating pattern & 0.251 & 0.935 & 0.246 & $<0.001$ \\
\hline & GAD-7 score & 0.336 & -0.270 & -0.363 & $<0.001$ \\
\hline & BFI extraversion & 0.409 & 0.918 & 0.172 & 0.012 \\
\hline & $\mathrm{BFI}$ agreeableness & 0.475 & 1.775 & 0.286 & $<0.001$ \\
\hline & Fitness test: $F=33.013, P<0.001$ & & & & \\
\hline \multirow[t]{9}{*}{ Environment } & Constant & & 3.654 & & \\
\hline & Education & 0.354 & 0.248 & 0.140 & 0.016 \\
\hline & Practice of religion & 0.476 & 0.314 & 0.141 & 0.014 \\
\hline & Active lifestyle & 0.538 & 0.715 & 0.241 & $<0.001$ \\
\hline & Duration of DM & 0.571 & -0.031 & -0.139 & 0.016 \\
\hline & BFI extraversion & 0.597 & 0.857 & 0.202 & 0.002 \\
\hline & $\mathrm{BFI}$ agreeableness & 0.614 & 1.562 & 0.327 & $<0.001$ \\
\hline & BFI neuroticism & 0.632 & -0.802 & -0.223 & 0.001 \\
\hline & Fitness test: $F=30.208, P<0.001$ & & & & \\
\hline
\end{tabular}

the strongest independent variable that affected the health related QOL and life satisfaction. Another study found that personality was a major determinant of QOL in patients with diabetes, largely through its effect on mediating variables such as coping style and mood (Rose et al. 1998).

In recent years, much research has focused on the potential role of neuroticism in patients with chronic illnesses such as DM. Neuroticism is defined as a "tendency to experience negative emotions" (McCrae \& Costa 
2008) and it is characterised by facets such as anxiety, angry hostility, and depression (John \& Srivastava 1999). In this current study, we found that elderly participants who scored higher on the trait of neuroticism were more likely to have a poorer physical QOL and poorer environment QOL. It is possible that this is because of neuroticism on mediating factors such as mood, behaviour, and coping strategy. For example, one study found that diabetic patients who scored higher on neuroticism were more likely to exhibit depressive symptoms and less likely to adhere to diabetes lifestyle management plans (Novak et al. 2017). Similarly, in a sample of patients with type 2 DM, it has been shown that higher levels of neuroticism were associated with greater chronic fatigue and reduced QOL (Momeniarbat et al. 2017). Furthermore, higher levels of neuroticism in diabetic adolescents were also associated with fewer proactive coping strategies and poorer psychological QOL (Kalka \& Karcz 2020).

On the contrary, we found that elderly participants who scored higher on the trait of conscientiousness were more likely to have a better physical QOL and a better psychological QOL. Conscientiousness is associated with facets such as self-discipline, competence, and dutifulness (John \& Srivastava 1999). Again, as was the case with neuroticism, it is possible that conscientiousness indirectly affects QOL through its effect on mediating factors, such as mood, behaviour, and coping strategy. For instance, one study found that in a sample of DM patients undergoing distress reduction interventions, those with greater conscientiousness were more likely to improve their medication adherence and reduce their disease-associated emotional burden (Fisher et al. 2014). Similarly, higher levels of conscientiousness have been associated with better diseaserelated self-care behaviours, such as better glucose monitoring (Skinner et al. 2014). Another study found conscientiousness to be associated with more effective coping strategies in patients with DM (Lawson et al. 2010). These findings can manifest clinically as well, as it has been shown that low levels of conscientiousness may contribute to a faster rate of renal failure among patients with DM (Brickman et al. 1996).

Finally, in this study, participants who scored higher on the trait of agreeableness were more likely to have a better social relationship QOL and a better environment QOL. Agreeableness is associated with facets such as altruism, tendermindedness, and trust (John \& Srivastava 1999). There has been less research centred around the trait of agreeableness in the context of DM. However, it has been found that the trait of agreeableness was associated with increased levels of seeking social support (Lawson et al. 2010). This could in part explain why we found there to be an association between the level of a patient's agreeableness and their social QOL.

This current study should be interpreted considering a few limitations. Firstly, the sample size used in this study was small and the 
study was conducted in a single tertiary healthcare referral centre in Kuala Lumpur, Malaysia. As such, the findings may not be generalisable to the entire population of elderly patients with diabetes. Further, due to the cross-sectional nature of the project, no causal relationships between any of the personality traits and any of the domains of QOL can be made. Social desirability bias might have affected responses to some of the questions, resulting in higher tendency to endorse health behaviours (e.g., active lifestyle) and reduced inclination to affirm unhealthy behaviours (e.g. alcohol and drug use). Future research should aim to involve multicentre subject recruitment of elderly diabetic patients to confirm this study's findings. Additionally, the mediating roles of mood and coping styles on the influence of personality traits on QOL in the patient population warrant further research.

\section{CONCLUSION}

Findings of the present study suggest that personality traits may play an important role in moderating the impact of DM on the lives of patients, even among elderly patients with DM, who are likely to have lived with the disease for a longer period. Traits such as conscientiousness and agreeableness may act as protective factors for the QOL in elderly patients with diabetes, whereas the trait of neuroticism could potentially be a risk factor for reduced QOL in these patients. Our study emphasises the important role of personality traits on the physical, psychological, social relationship, and environmental QOL of an elderly patient with DM. Health care providers should be aware of the various psychosocial factors, particularly the personality traits, that can contribute to a patient's illness experience. By recognising how a patient's personality may contribute to their ability to manage and cope with their disease, health care providers will be better equipped to provide quality care to each of their patients.

\section{ACKNOWLEDGEMENT}

The authors would like to thank Dr Nurul Hazwani Hatta, Dr Puteri Arnawati, and Dr Amelia Yasmin Zulkifli for their involvement in data collection for this study. This work was funded by the Young Lecturers Incentive Grant (GGPM-2019-024) from the Research University Fund of the National University of Malaysia, as well as the University of Toronto Comprehensible Research Experience for Medical Students (CREMS) and Medical Alumni Association (MAA).

\section{REFERENCES}

Beck, A.T., Steer, R.A., Brown, G.K. 1996. Manual for the Beck Depression Inventory-II. San Antonio, TX: Psychological Corporation.

Brickman, A.L., Yount, S.E., Blaney, N.T., Rothberg, S.T., De-Nour, A.K. 1996. Personality traits and long-term health: Status the influence of neuroticism and conscientiousness on renal deterioration in type-1 diabetes. Psychosomatics 37(5): 459-68.

Cobb-Clark, D.A., Schurer, S. 2012. The stability of big-five personality traits. Econ Lett 115(1): 11-5.

Costa, P.T. Jr., McCrae, R.R. 1990. Personality disorders and the five-factor model of personality. J Pers Disord 4(4): 362-71.

Department of s Malaysia. 2016. Population 
projection (Revised) 2010-2040. https://www. dosm.gov.my/v1/index.php? r=column/pdfPre v\&id=Y3kwU2tSNVFDOWp1YmtZYnhUeVBE dz09 [27 Feb 2020].

Elran-Barak, R., Weinstein, G., Beeri, M.S., RavonaSpringer, R. 2019. The associations between objective and subjective health among older adults with type 2 diabetes: The moderating role of personality. J Psychosom Res 117: 41-7.

Fisher, L., Hessler, D., Masharani, U., Strycker, L. 2014. Impact of baseline patient characteristics on interventions to reduce diabetes distress: the role of personal conscientiousness and diabetes self-efficacy. Diabet Med 31(6): 739-746.

Fowler, M.J. 2008. Microvascular and macrovascular complications of diabetes. Clin Diabet 26(2):7782.

Gregg, E.W., Engelgau, M.M., Narayan, V. 2002. Complications of diabetes in elderly people: Underappreciated problems include cognitive decline and physical disability. Brit Med J 325(7370): 916-17.

Hasanah, C.I., Naing, L., Rahman, A.R. 2003. World Health Organization Quality of Life Assessment: Brief Version in Bahasa Malaysia. Med J Malaysia 58(1): 79-88.

Hussein, Z., Taher, S.W., Gilcharan Singh, H.K, Chee Siew Swee, W. 2015. Diabetes care in Malaysia: problems, new models, and solutions. Ann Glob Health 81(6): 851-62.

Imayama, I., Plotnikoff, R.C., Courneya, K.S., Johnson, J.A. 2011. Determinants of quality of life in type 2 diabetes population: the inclusion of personality. Qual Life Res 20(4): 551-8.

John, O.P., Donahue, E.M., Kentle, R.L. 1991. The Big Five Inventory-Versions $4 \mathrm{a}$ and 54. Berkeley, CA: University of California, Berkeley, Institute of Personality and Social Research.

John, O.P., Srivastava, S. 1999. The Big Five trait taxonomy: History, measurement, and theoretical perspectives. In Handbook of Personality: Theory and Research. 2nd edition. Edited by Pervin LA, John OP. New York: Guilford Press; 102-38.

Kalka, D., Karcz, B. 2020. Personality, coping with stress and quality of life in individuals at risk of type 2 diabetes in late adolescence-mediation model testing. Eur J Dev Psychol 17(2): 165-78.

Lawson, V.L., Bundy, C., Belcher, J., Harvey, J. 2010. Mediation by illness perceptions of the effect of personality and health threat communication on coping with the diagnosis of diabetes. $\mathrm{Br}$ J Health Psychol 15(3): 623-42.

McCrae, R.R., Costa, P.T. Jr. 2008. The fivefactor theory of personality. In Handbook of Personality: Theory and Research. Edited by John OP, Robins RW, Pervin LA. New York: Guilford Press; 159-81.

Sidik, S.M, Arroll, B., Goodyear-Smith, F. 2012.
Validation of the GAD-7 (Malay version) among women attending a primary care clinic in Malaysia. J Prim Health Care 4(1): 5-11.

Momeniarbat, F., Karimi, J., Erfani, N., Kiani, J. 2017. The role of neuroticism and psychological flexibility in chronic fatigue and quality of life in patients with type 2 diabetes. Rom J Diabetes Nutr Metab Dis 24(2): 137-48.

Muhammad, H., Roodenburg, J., Moore, D. W. 2018. The adaptation of the Big Five Inventory in measuring Malaysian youths' personality traits. Int J Adv App/ Sci 5(7): 8-14.

Mukhtar, F., Oei, T.P.S. 2008. Exploratory and confirmatory factor validation and psychometric properties of the Beck Depression Inventory for Malays (BDI Malay) in Malaysia. MJP Online Early 17(1). http://www.psychiatrymalaysia.org/ file_dir/16510073164861a7e47a8ae.pdf [13 Jan 2019].

Novak, J.R., Anderson, J.R., Johnson, M.D., Hardy, N.R., Walker, A., Wilcox, A., Lewis, V.L., Robbins, D.C. 2017. Does personality matter in diabetes adherence? Exploring the pathways between neuroticism and patient adherence in couples with type 2 diabetes. Appl Psychol Health Well Being 9(2): 207-27.

Rose, M., Burkert, U., Scholler, G., Schirop, T., Danzer, G., Klapp, B.F. 1998. Determinants of the quality of life of patients with diabetes under intensified insulin therapy. Diabetes Care 21(11): 1876-85.

Schimmack, U., Oishi, S., Furr, R.M., Funder, D.C. 2004. Personality and life satisfaction: A facetlevel analysis. Pers Soc Psychol Bull 30(8): 1062-75.

Skinner, T., Bruce, D.G., Davis, T., Davis, W.A. 2014. Personality traits, self-care behaviours and glycaemic control in Type 2 diabetes: The Fremantle Diabetes Study Phase II. Diabet Med 31(4): 487-92.

Soldz, S., Vaillant, G.E. 1999. The Big Five personality traits and the life course: A 45-year longitudinal study. J Res Pers 33(2): 208-32.

Spitzer, R.L., Kroenke, K., Williams, J.B., Löwe, B. 2006. A brief measure for assessing generalized anxiety disorder: the GAD-7. Arch Intern Med 166(10): 1092-7.

WHOQOL Group. 1998a. The World Health Organization quality of life assessment (WHOQOL): development and general psychometric properties. Soc Sci Med 46(12): 1569-85.

WHOQOL Group. 1998b. Development of the World Health Organization WHOQOL-BREF quality of life assessment. Psychol Med 28(3): 551-8.

Yamaoka, K., Shigehisa, T., Ogoshi, K., Haruyama, K., Watanabe, M., Hayashi, F., Hayashi, C. 1998. Health-related quality of life varies with 
personality types: a comparison among cancer patients, non-cancer patients and healthy individuals in a Japanese population. Qual Life $\operatorname{Res}$ 7(6): 535-44.

Received: 21 Sept 2020

Accepted: 19 Feb 2021 